Original Article

\title{
Point-of-Care Ultrasound Training Among Intensivists in Singapore: A Multicentre Survey
}

Yie Hui Lau, ${ }^{1}$ MBBS, MMed (Anaes), FANZCA, Chee Hong Loh, ${ }^{2}$ MD, Board Cert (Int Med, Pulm Disease, Crit Care Med), FCCP, Wee Kim Fong, ${ }^{3}$ MBBS, MMed (Anaes), Shahla Siddiqui, ${ }^{4}$ MBBS, D ABA, FCCM, Chee Keat Tan, ${ }^{5}$ MD (UKM), MMed (Anaes), FAMS (Anaes, Intensive Care), Jonathan JE $\underline{\text { Tan }},{ }^{6}$ MBBS, MMed (Anaes), FANZCA, Claudia JC $\underline{\text { Tien, }},{ }^{7}$ MBBS, MMed (Anaes), FANZCA, Kay Choong See, ${ }^{8}$ MBBS, MRCP(UK), FCCP

\begin{abstract}
Introduction: Point of care ultrasound (POCUS) has become essential in the management of critically ill patients. Multiple POCUS training courses exist, but are not widely adopted in Singapore. Given the discordance between training supply and demand, we aimed to uncover the learning needs and barriers among intensive care medicine (ICM) doctors locally. Materials and Methods: An anonymous online survey was carried out from January to April 2019 among ICM doctors from 6 major teaching hospitals in Singapore. Results: 66 out of 160 ICM specialists and trainees responded

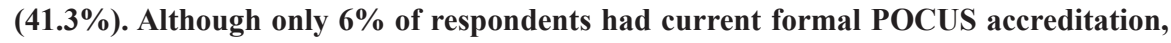
the majority were already using critical care echocardiography (CCE) and pleural/ lung ultrasound. $93 \%$ supported having a local training programme. More than $50 \%$ of trainees wanted training in CCE, diaphragm/muscle ultrasound, airway ultrasound and ultrasound for neurological disease. More than $\mathbf{5 0 \%}$ of specialists wanted training in diaphragm/muscle ultrasound, with mixed interests in other topics. The top 2 obstacles hindering POCUS training were lack of supervisors and locally based programmes. Conclusions: ICM doctors who responded were supportive of a local POCUS training programme. The programme's curriculum should address the learning needs of the majority of learners, with diaphragm/muscle ultrasound being the top unmet need. The programme would need to develop supervisors and be as affordable as possible.
\end{abstract}

Ann Acad Med Singap. 2020;49:630-42

Key words: Continuing medical education, Cross-sectional survey, Intensive Care, Ultrasound

\section{Introduction}

Point-of-care ultrasound (POCUS) has become an essential tool for managing critically ill patients. Systematic application of POCUS techniques such as critical care echocardiography (CCE) and lung ultrasound may change or confirm a diagnosis in more than $80 \%$ of cases, decrease utilisation of chest X-ray and computed tomography scans, reduce mechanical ventilation duration and assist with haemodynamic optimisation and intravenous fluid titration. ${ }^{1,2}$ In cardiac arrest situations, protocolised focused ultrasound provided diagnostic information and altered management in $78 \%$ of patients. ${ }^{3}$

\footnotetext{
${ }^{1}$ Department of Anaesthesiology, Intensive Care and Pain Medicine, Tan Tock Seng Hospital, Singapore

${ }^{2}$ Department of Respiratory and Critical Care Medicine, Changi General Hospital, Singapore

${ }^{3}$ Department of Anaesthesiology, Intensive Care and Pain Medicine, Tan Tock Seng Hospital, Singapore

${ }^{4}$ Department of Anaesthesia, Critical Care and Pain Medicine, Beth Israel Deaconess Medical Center, Boston, United States

${ }^{5}$ Department of Intensive Care Medicine, Ng Teng Fong General Hospital, Singapore

${ }^{6}$ Mount Elizabeth Novena Hospital, Singapore

${ }^{7}$ Department of Surgical Intensive Care, Division of Anaesthesiology and Perioperative Medicine, Singapore General Hospital, Singapore

${ }^{8}$ Division of Respiratory and Critical Care Medicine, National University Hospital, Singapore

Address for Correspondence: Dr Yie Hui Lau, Department of Anaesthesiology, Intensive Care and Pain Medicine, Tan Tock Seng Hospital, 11 Jalan Tan Tock Seng, Singapore 308433, Singapore

Email: yie_hui_lau@ttsh.com.sg
} 
Transthoracic echocardiography (TTE) and general ultrasound have traditionally been done, respectively, by cardiologists and diagnostic radiologists. Several drawbacks of the traditional arrangement exist. Firstly, ultrasound scans are often not contemporaneous and cannot help answer urgent clinical questions or directly influence the immediate treatment. Secondly, ultrasound image acquisition and reporting are separated, which hinders clinical correlation. Thirdly, it is not easily repeatable and is not suitable for tracking therapeutic responses or rapid clinical changes. Given the drawbacks of the traditional arrangement, managing intensivists should learn and apply POCUS at the bedside. International experts agree that general POCUS and basic CCE should be mandatory in the ICM training curriculum. ${ }^{4}$ Worldwide, assessment tools for CCE, ${ }^{5}$ and guidelines for training and competency in POCUS ${ }^{6,7}$ have been developed. However, no standardised curriculum has been universally adopted. ${ }^{8}$ For instance, a comparison of POCUS training programmes in Europe showed that there was a lack of agreement among five accreditation programmes. ${ }^{9}$

Multiple courses and postgraduate programmes for POCUS training exist, such as the World Interactive Network Focused On Critical UltraSound (WINFOCUS), Rapid Assessment by Cardiac Echo (RACEplus) or Certificate in Clinician Performed Ultrasound (CCPU) by the Australasian Society for Ultrasound in Medicine (ASUM), but none has been widely adopted in Singapore. Given the discordance between POCUS training supply and demand, perhaps, the learning needs or barriers have not been fully addressed. National surveys on POCUS in intensive care units (ICU) have been conducted in Australia, ${ }^{10}$ Brazil $^{11}$ and in the United States. ${ }^{12}$ However, it is uncertain if results from the preceding 3 national surveys can be generalised globally, and there has been no survey done in Asia. As part of the Society of Intensive Care Medicine (Singapore)'s effort to develop a national curriculum for POCUS, we embarked on a survey among practising intensivists and trainees. In this survey, we aimed to study the current practice of POCUS, availability of training and resources, and the perceived needs for future training and competency assessment.

\section{Materials and Methods}

\section{Data Collection}

Ethics approval was exempted by the National Healthcare Group-Domain Specific Review Board (2018/01134). Thereafter, we conducted an anonymous online survey of intensive care medicine (ICM) specialists and trainees in all 6 major teaching hospitals of Singapore. "Specialists" were defined as practising intensive care physicians who were accredited by the Singapore Specialist Accreditation Board and registered as such by the Singapore Medical Council. "Trainees" were defined as doctors who were undergoing specialist training in intensive care and had yet to complete the local exit examination. Data was collected between January and April 2019. A web-based online survey platform (SurveyMonkey ${ }^{\mathrm{TM}}$ ) was used. This platform was accessible only by the principal investigator, and the data were anonymised and encrypted. No financial support was received from any source. Participants were emailed the invitation to the survey via members of the study team, who were representatives of the ICUs in their respective hospitals. Reminders were sent via email and phone about 1 and 3 months after the initial invitation. Participants did not receive any renumeration for their participation.

\section{Survey Construction}

All members of the study team were certified intensive care specialists who perform and teach POCUS. The survey was primarily designed by 2 authors with every member of the study team providing feedback. Using a consensus approach, there were 3 rounds of email discussions and one in-person meeting to select the final 50 questions (see Supplement 1). The survey was then divided into 7 sections:

(1) Demographics and characteristics of respondents

(2) Current resources and usage

(3) Individual practice

(4) Individual training in POCUS

(5) Perceived obstacles

(6) Willingness to pay

(7) Future practice and formal competency assessment needs

Sections of the survey pertaining to individual practice were designed with a 4-point Likert scale to assess frequency of performing POCUS or the level of agreement to statements, such as "daily", "several times a week", "hardly ever or never", "strongly disagree", "disagree", "agree and "strongly agree".

Abdominal ultrasound included FAST (Focused Abdominal Sonography in Trauma). Ultrasound for neurological disease included ultrasound to assess optic nerve sheath diameter (ONSD) or cerebral circulation. Ultrasound for vascular diagnostics included screening for abdominal aortic aneurysm or deep vein thrombosis. 


\section{Statistical Analysis}

Categorical variables were presented as absolute numbers and percentages. Where statistical analysis to compare percentages was done, Fisher's exact test was used. Thematic analysis was done by the first and last authors using the free text comments for the following question: "Any other ideas on how to implement critical care ultrasound training". Missing data were not imputed.

\section{Results}

Demographics and Characteristics of Study Respondents

One hundred and sixty ICM specialists and trainees received the survey invitation, and 66 responded (response rate $41.3 \%$ ) (Table 1). 57.6\% of respondents were male, and the mean age was 38.8 years (standard deviation 7.3 years). Of the respondents, $13(19.7 \%)$ were trainees and $53(80.3 \%)$ were specialists. $11(18 \%)$ respondents were participating in POCUS training programmes as faculty.

\section{Current Resources and Usage}

All 66 respondents had access to an ultrasound machine dedicated for use in the ICU (Table 2). 57 $(89 \%)$ were familiar with the machines available in their department. For the majority of POCUS scans, the respondents did not record static, cine clips or reports in the electronic health records. Specifically, if recorded, 28 (42.4\%) would only store POCUS reports, 36 (54.5\%) static clips and $36(54.5 \%)$ cine images in the ultrasound machine and not in picture archiving and communication systems (PACS), paper or electronic medical records. Thirteen (19.7\%) reported that they would also save POCUS reports in electronic health records.

\section{Individual Practice}

All 66 respondents used real-time ultrasound to guide central venous catheter (CVC) insertion. Ultrasound for vascular access, CCE, and pleural/lung ultrasound were the most common types of POCUS scans done (Figure 1). For new admissions, the commonest

Table 1. Characteristics of Respondents

\begin{tabular}{|c|c|}
\hline & All respondents $n=66(\%)$ \\
\hline \multicolumn{2}{|l|}{ Years after graduation from basic medical degree } \\
\hline 1 to 5 & $3(4.5)$ \\
\hline 6 to 10 & $21(31.8)$ \\
\hline 11 to 15 & $15(22.7)$ \\
\hline 16 to 20 & $11(16.7)$ \\
\hline 21 to 25 & $12(18.2)$ \\
\hline 26 to 30 & $3(4.5)$ \\
\hline 31 to 35 & $1(1.5)$ \\
\hline \multicolumn{2}{|l|}{ Years since obtaining Intensive Care qualifications } \\
\hline 1 to 5 & $23(34.8)$ \\
\hline 6 to 10 & $10(15.2)$ \\
\hline 11 to 15 & $10(15.2)$ \\
\hline 16 to 20 & $4(6.1)$ \\
\hline Not applicable (currently still training) & $19(28.8)$ \\
\hline \multicolumn{2}{|l|}{ Primary (base) specialty } \\
\hline Anaesthesiology & $31(47.0)$ \\
\hline Respiratory Medicine & $28(42.4)$ \\
\hline General Medicine & $6(9.1)$ \\
\hline Emergency Medicine & $1(1.5)$ \\
\hline \multicolumn{2}{|c|}{ Completed an official ICM fellowship, local or overseas } \\
\hline Yes & $40(60.6)$ \\
\hline No & $7(10.6)$ \\
\hline Not applicable (currently training) & $19(28.8)$ \\
\hline \multicolumn{2}{|l|}{ Types of ICUs } \\
\hline Mixed medical and Surgical & $13(20.3)$ \\
\hline Medical & $27(42.2)$ \\
\hline Surgical (general surgical, burns, neurosurgical) & $24(37.5)$ \\
\hline
\end{tabular}

ICM: Intensive Care Medicine, ICU: Intensive Care Unit 
Table 2. Current POCUS Resources

\begin{tabular}{ll}
\hline Resources & $\mathrm{n}=64(\%)^{*}$ \\
\hline Ultrasound machine dedicated for use in the ICU & $0(0)$ \\
No & $1(1.5)$ \\
Yes, but unsure about specifications & $8(12.5)$ \\
Yes, without continuous and pulse-wave doppler imaging & $27(42.2)$ \\
Yes, with continuous, pulse-wave, but not tissue doppler & $29(45.3)$ \\
Yes, with continuous, pulse-wave and tissue- doppler & $2(3.1)$ \\
Not answered & $28(42.4)$ \\
Payoffs for POCUS scans & $13(19.7)$ \\
None & $0(0)$ \\
Only for institution or unit & $5(7.6)$ \\
Only for physician & $18(27.3)$ \\
For both institution or unit and physician & \\
Unknown & \\
\hline
\end{tabular}

$* \mathrm{n}=2$ missing data

ICU: Intensive care unit

POCUS: Point-of-Care ultrasound

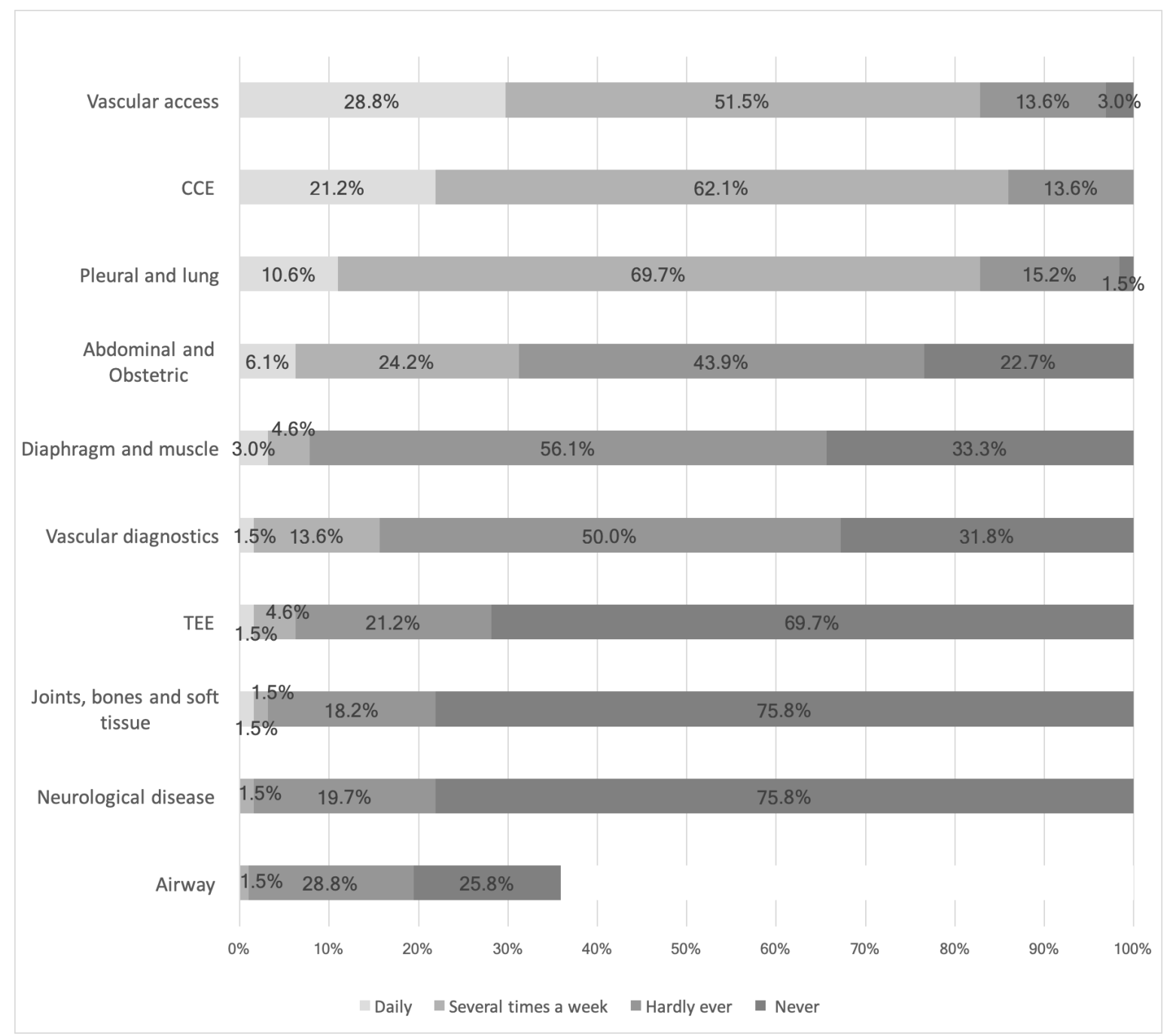

Fig 1. Most Common Types of POCUS Scans 
POCUS performed was CCE $(60 / 66,90.9 \%)$ and pleural/lung ultrasound $(52 / 66,78.8 \%)$. Three $(4.5 \%)$ responded that they did not routinely perform POCUS of any form for new admissions. Sixty-one (92.4\%) agreed that the application of POCUS frequently changed the real-time management of ICU cases under their care; the $5(7.6 \%)$ respondents who disagreed were all specialists. Prior to performing a percutaneous tracheostomy, among 31 respondents, 19 (61.3\%) reported doing airway ultrasound routinely.

\section{Individual Point-of-Care Ultrasound Training}

POCUS or CCE courses attended by respondents included World Interactive Network Focused On Critical UltraSound (WINFOCUS) course $(n=24)$, RACEplus course $(\mathrm{n}=23)$, Basic Assessment and Support in Intensive Care (BASIC) Transthoracic echocardiography course $(n=18)$, the National Heart Centre Coronary Care Unit Symposium $(n=5)$, and pre-conference workshops of international ICM congresses $(n=27)$.

Twenty-one out of $66(32 \%)$ respondents had previous formal full TTE training, defined as having direct supervision for a stipulated minimum number of full TTE scans, by an accredited supervisor/ sonographer. The commonest type of POCUS respondents received formal training in was CCE 42/64 (63.6\%), followed by pleural/lung ultrasound 27/64 (40.9\%), ultrasound for vascular access 21/64 (31.8\%) and FAST 15/64 (22.7\%). Less than $10 \%$ of respondents said they received training in each of the other types of POCUS (transoesophageal echocardiography (TEE), airway, abdominal/ obstetric, diaphragm/muscle, ultrasound for neurological diseases). None received training in joint/bone ultrasound.

Only $7(6 \%)$ out of 62 respondents had current POCUS accreditation, such as Diploma in Clinical Ultrasound, European Association of Echocardiography/ European Association of Cardiovascular Imaging (EAE/ EACVI) TEE, Focused Intensive Care Echocardiography (FICE), or Postgraduate Certificate in Clinical Ultrasound. Two out of $66(3 \%)$ answered that formal renewal of credentialing in POCUS was required by their institution. The median number of full TTE scans done by the respondents was 0 (range 0 to $>100$ ). In contrast, the median number of CCE scans done was 40 (range 0 to $>100$ ). Within the scope of CCE, $80.6 \%$ and $74.2 \%$ of respondents were comfortable with image acquisition and image interpretation, respectively. With regard to POCUS (including other types of scans, not just limited to CCE), $85.5 \%$ of respondents were confident with POCUS with clinical management. Fifteen (22.7\%) stated that there was no need for competency assessment for management at the bedside.

\section{Perceived Obstacles}

The survey included a section on what respondents perceived as obstacles to advancing their own training in POCUS, and to explore the challenges that may arise in a future training programme. Of the 66 responses, the commonest obstacles reported were "lack of supervisors" ( $\mathrm{n}=59,89.4 \%)$, "programmes are only available overseas" $(\mathrm{N}=39,59.1 \%)$, "lack of storage for clips and images" $(\mathrm{n}=20,30.3 \%)$," lack of time to scan" $(\mathrm{n}=20,30.3 \%)$," medico-legal implications" $(20 / 66,30.3 \%)$ and "inadequate knowledge" $(n=18$, $27.3 \%$ ). Free text comments given were: "lack of time away from clinical and administrative work", "lack of dedicated framework to allow to work towards an external accreditation" and "to be certified, need for adequate supervisor framework to log scans and more importantly to teach and improve technique".

\section{Willingness to Pay}

For initial training and competency assessment, assuming it takes one year, respondents were willing to commit a median of 50 Singapore dollars (SGD) (range SGD0-200). The median number of hours per month a learner can commit to training was 12 hours (range 1 to 60 hours). For re-accreditation, assuming it is needed every 5 years, the median sum respondents were willing to commit was SGD32 (range SGD0-478).

\section{Future Training and Competency Assessment Needs}

Fifty-three respondents (93\%) agreed that there was a need for a local POCUS training programme. Fifty-seven $(72 \%)$ respondents agreed that there was a need for formal competency assessment in POCUS, while 4 (7\%) disagreed.

Regarding what respondents would like to receive formal training for, the top 2 choices overall were CCE $(n=38)$, and diaphragm/muscle ultrasound $(n=28)$. There were differences in what trainees and specialists wanted to receive for training (Table 3). Regarding which areas of POCUS respondents would like to receive formal competency assessment for, the top 3 choices were CCE $(\mathrm{n}=46)$, pleural/lung ultrasound $(\mathrm{n}=29)$ and vascular diagnostics $(\mathrm{n}=20)$.

\section{Thematic Analysis on How to Implement POCUS Training}

When surveyed on the preferred platforms for acquiring content knowledge, the majority indicated 
Table 3. Requests for formal training

\begin{tabular}{|c|c|c|c|c|}
\hline & $\begin{array}{l}\text { All } \\
n=66(\%)\end{array}$ & $\begin{array}{l}\text { Trainee } \\
n=19(\%)\end{array}$ & $\begin{array}{l}\text { Specialist } \\
\mathrm{n}=47(\%)\end{array}$ & P-value \\
\hline $\mathrm{CCE}$ & $38(57.6)$ & $17(89.5)$ & $21(44.7)$ & 0.001 \\
\hline Diaphragm and muscle ultrasound & $38(57.6)$ & $14(73.7)$ & $24(51.1)$ & 0.108 \\
\hline Airway ultrasound & $27(40.9)$ & $12(63.2)$ & $15(31.9)$ & 0.028 \\
\hline Ultrasound for Neurological disease & $27(40.9)$ & $12(63.2)$ & $15(31.9)$ & 0.028 \\
\hline TEE & $25(38.9)$ & $5(26.3)$ & $20(42.6)$ & 0.270 \\
\hline Abdominal or obstetric ultrasound & $24(36.4)$ & $9(47.4)$ & 15 (31.9) & 0.268 \\
\hline Pleural and lung ultrasound & $24(36.4)$ & $11(57.9)$ & $13(27.7)$ & 0.027 \\
\hline Ultrasound for vascular access & $12(18.2)$ & $5(26.3)$ & $7(14.9)$ & 0.304 \\
\hline
\end{tabular}

CCE: Critical care echocardiography

TEE: Transesophageal echocardiography

that they preferred in-person lectures $(40 / 66,60.6 \%)$, web-based lectures $(35 / 66,53.0 \%)$, internetbased applications $(26 / 66,39.4 \%)$ and YouTube ${ }^{\mathrm{TM}}$ $(17 / 66,25.8 \%)$. Only 1 respondent opted for social media such as Facebook ${ }^{\mathrm{TM}}$ or Twitter ${ }^{\mathrm{TM}}$. Other respondents $(7 / 66,12.2 \%)$ suggested learning from books and on-the-job training. Thematic analysis was done for the qualitative free text comments on how to implement POCUS training, revealing themes associated with accessibility, curriculum and credibility (Table 4).

\section{Discussion}

\section{Key Findings}

One hundred and sixty ICM specialists and trainees received the survey invitation, and 66 responded $(41.3 \%)$. Although only $6 \%$ of respondents had current formal POCUS accreditation, the majority of ICM doctors were already using it frequently. Ninety three percent supported having a local training programme. Additional POCUS training requested but not frequently available now were TEE, airway and diaphragm/muscle ultrasound. More than $50 \%$ of trainees wanted training in CCE, diaphragm/muscle ultrasound, airway ultrasound, and ultrasound for neurological disease. More than $50 \%$ of specialists wanted training in diaphragm/muscle ultrasound, with mixed interests in other topics. Training in ultrasound for vascular access was not highly requested, likely because it is part of base-specialty training (such as in Anaesthesiology or Respiratory Medicine), prior to entering ICM training. The top 2 obstacles hindering formal POCUS training were lack of supervisors and locally based programs.

\section{Relation to Previous Literature}

All respondents had access to an ultrasound machine dedicated for use in the ICU, which is more than ICUs in Brazil, $64 \%{ }^{11}$ and $83-95 \%$ in USA. ${ }^{12}$ All the respondents used real-time ultrasound for $\mathrm{CVC}$ insertion, which is in line with international guidelines. ${ }^{13} 85 \%$ and $83 \%$ of respondents here reported using CCE and lung ultrasound, respectively, at least several times a week during their ICU practice, which is similar to ICUs in Australia (94\% and $83 \%$ respectively). ${ }^{10}$ The 3 most common POCUS modalities in ICUs locally (CCE, lung ultrasound and ultrasound for CVC insertion) were slightly different from USA (procedural guidance, vascular access, CCE) ${ }^{12}$ or Brazil where lung ultrasound was only performed by $8.5 \% .{ }^{11}$

The majority of respondents had exposure to 1 to 2-day training courses, but far fewer had exposure to formal training programmes, board examinations or o verseas diploma programmes with remote supervision. This is unlike respondents from Australian ICUs who predominantly had training through courses by ASUM or diploma courses,,$^{10}$ or USA, who had training through national programmes. ${ }^{12}$ 
Table 4. Thematic analysis for comments on "any other ideas on how to implement POCUS training"

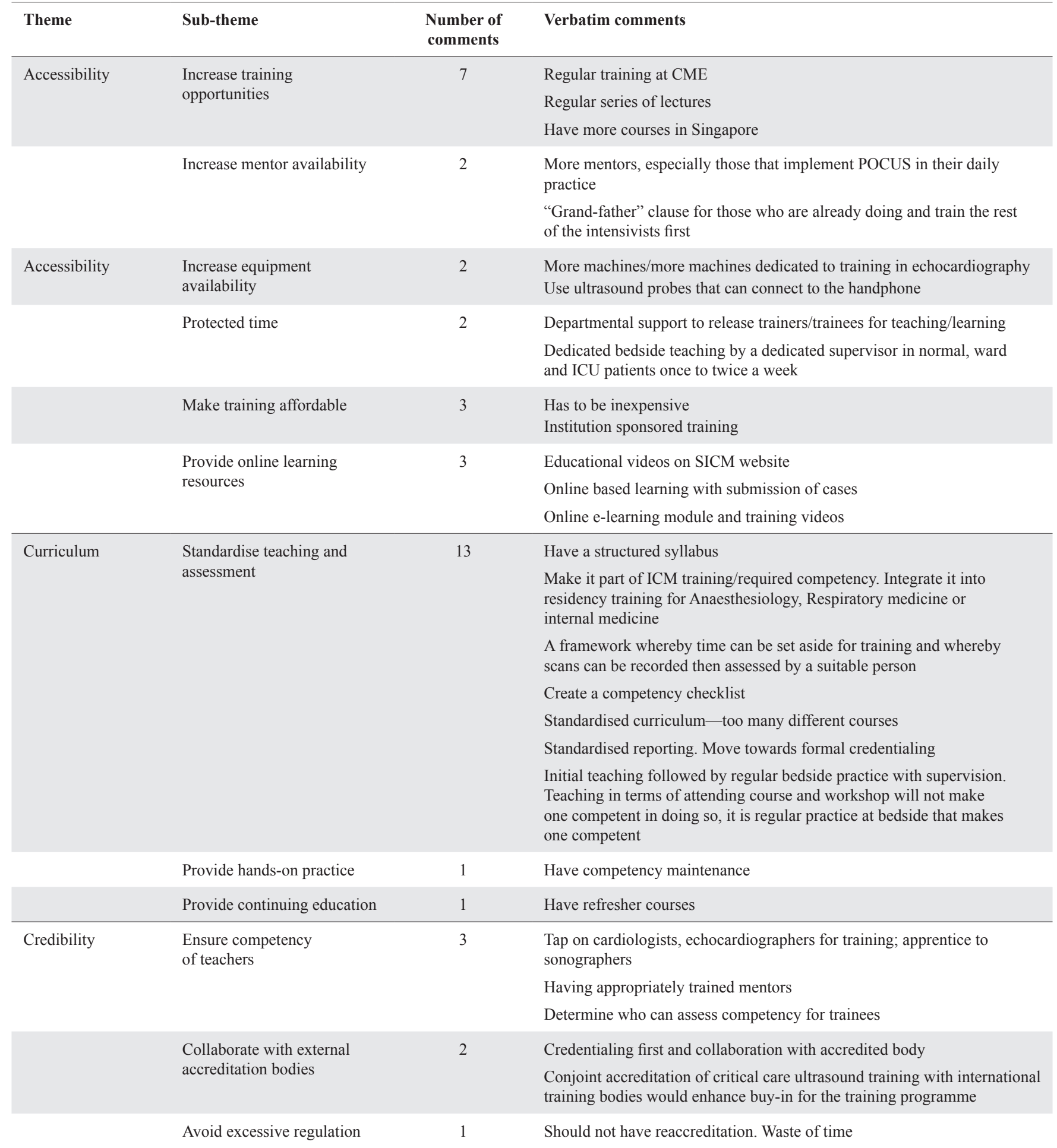

CME: Continuing Medical Education; ICU: Intensive Care Unit; ICM: Intensive Care Medicine; POCUS: Point-of-care Ultrasound; SICM: Society of Intensive Care Medicine (Singapore) 
The majority of respondents stated "lack of standardised competencies", "lack of supervisors" and "training programmes are only available overseas" as the top barriers to embarking on formal training. This is similar to studies done in other regions. ${ }^{9,10,15,16}$ Locally, among internal medicine physicians, lack and cost of an ultrasound machine were also significant barriers to learning. ${ }^{14}$ Importantly, lack of equipment ${ }^{14,16}$ or resistance from other ultrasound providers ${ }^{10}$ did not appear as one of the barriers in this survey. Lengthy and costly overseas programmes may not be necessary for competency in specific areas of POCUS, ${ }^{6}$ pleural and lung ultrasound ${ }^{17}$ or CCE. ${ }^{18,19}$ Local learners want to do things cheaply and quickly, not aligning with most international curricula.

\section{Study Implications}

Currently in Singapore, POCUS training is not mandatory for specialty accreditation in ICM. Nonetheless, almost all respondents agreed there is a need for a local POCUS training programme and formal competency assessment, which is in agreement with international statements on training standards for POCUS in ICU. ${ }^{4}$

More respondents were comfortable with image acquisition than with image interpretation for $\mathrm{CCE}$, which confirmed that $\mathrm{CCE}$ needs special attention to training, as it has been shown that individual domains of CCE are picked up differentially. ${ }^{18}$

While overall learning needs may be similar across various populations, some local differences would exist. ${ }^{14}$ Hence, training cannot depend entirely on a common global curriculum; local modifications and enhancements would be necessary.

Respondents' suggestions on ways to improve POCUS training locally included: having a structured curriculum; and, after having attended courses, having continued supervision by trained mentors in the hospital. Respondents still wanted in-person lectures and teaching. Given the above, web-based teaching will not fulfil learning needs. Teaching and faculty development need to be done locally. Respondents asking for protected time reflects the need for POCUS training at the bedside to be valid and to maximise benefits to learners, as outlined by Sewa and Anantham. ${ }^{20}$ To increase validity, we will need to ensure that trainers and trainees both have some protected teaching time.

The survey also showed that most respondents were willing to pay for training and competency assessment, which can inform future financing models for ultrasound training.
In the ICU, POCUS is used to complement physical examination at the bedside, rather than as a standalone radiological tool. This is especially true for resourcelimited settings with lower-end ultrasound systems, without storage capability or central archiving systems, which is not in alignment with most international curricula. Even in a resource-rich setting like Singapore, we found that the majority of POCUS clips were not reported or recorded in electronic health records. Although this has its drawbacks, such as the inability to compare images or clips, it reflects the reality of POCUS practice. As such, for training to succeed in an authentic clinical environment, supervision and learning needs to be like teaching bedside physical examination and storage needs to be point-of-care.

\section{Strengths and Limitations}

This is the first nation-wide survey in an Asian country on the practice of POCUS amongst ICM physicians. The limitations of this study include a lowmoderate response rate of $41.3 \%$, which may affect the representativeness of the results. However, this is comparable to email survey response rates in other medical publications. ${ }^{21}$ Participation bias is possible as physicians who use POCUS regularly may be keener to participate in the survey. Based on this survey, ICM specialists appear to want a programme, but with only $19.7 \%$ of respondents being ICM trainees, the sample may be too small to confidently determine their interest.

\section{Conclusions}

Our survey showed that ICM physicians who responded were supportive of a local POCUS training programme. The programme's curriculum should include modules that address the learning needs for the majority of trainees and intensivists, diaphragm/muscle ultrasound being the top unmet need. Additionally, the programme would need to develop supervisors and be as affordable as possible.

\section{REFERENCES}

1. Pontet J, Yic C, Díaz-Gómez JL, Rodriguez P, Sviridenko I, Méndez $\mathrm{D}$, et al. Impact of an ultrasound-driven diagnostic protocol at early intensive-care stay: a randomized-controlled trial. Ultrasound J. 2019 Sep 30;11(1).

2. Zieleskiewicz L, Muller L, Lakhal K, Meresse Z, Arbelot C, Bertrand P-M, et al. Point-of-care ultrasound in intensive care units: assessment of 1073 procedures in a multicentric, prospective, observational study. Intensive Care Med 2015 Sep;41(9):1638-47.

3. Breitkreutz R, Price S, Steiger H V., Seeger FH, Ilper H, Ackermann $\mathrm{H}$, et al. Focused echocardiographic evaluation in life support and peri-resuscitation of emergency patients: A prospective trial. Resuscitation. 2010 Nov;81(11):1527-33. 
4. Cholley BP. International expert statement on training standards for critical care ultrasonography. Intensive Care Med. 2011 Jul; 37(7):1077-83

5. Millington SJ, Arntfield RT, Hewak M, Hamstra SJ, Beaulieu Y, Hibbert $\mathrm{B}$, et al. The rapid assessment of competency in echocardiography scale. J Ultrasound Med. 2016 Jul;35(7):1457-63.

6. Arntfield RT, Millington SJ, Ainsworth CD, Arora RC, Boyd J, Finlayson $\mathrm{G}$, et al. Canadian recommendations for critical care ultrasound training and competency. Can Respir J. November/ December 2014;21(6):341-345.

7. Vieillard-Baron A, Mayo PH, Vignon P, Cholley B, Slama M, Pinsky MR, et al. International consensus statement on training standards for advanced critical care echocardiography. Intensive Care Medicine. 2014 May;40(5):654-66.

8. Mayo PH, Beaulieu Y, Doelken P, Feller-Kopman D, Harrod C, Kaplan A, et al. American college of chest physicians/ la societédé réanimation de langue française statement on competence in critical care ultrasonography. Chest. 2009 Apr 1;135(4):1050-60.

9. Galarza L, Wong A, Malbrain MLNG. The state of critical care ultrasound training in Europe: A survey of trainers and a comparison of available accreditation programmes. Anaesthesiol Intensive Ther. 2017;49(5):382-6.

10. Yang Y, Royse C, Royse A, Williams K, Canty D. Survey of the training and use of echocardiography and lung ultrasound in Australasian intensive care units. Crit Care 2016 Oct 24;20(1):339.

11. Pellegrini JAS, Cordioli RL, Grumann ACB, Ziegelmann PK, Taniguchi LU. Point-of-care ultrasonography in Brazilian intensive care units: a national survey. Ann Intensive Care. 2018 Apr 20;8(1):50.

12. Mosier JM, Malo J, Stolz LA, Bloom JW, Reyes NA, Snyder LS, et al. Critical care ultrasound training: A survey of US fellowship directors. J Crit Care. 2014 Aug;29(4):645-9.

13. Practice Guidelines for Central Venous Access 2020: An Updated Report by the American Society of Anesthesiologists Task Force on Central Venous Access*. Anesthesiol J Am Soc Anesthesiol. 2020 Jan $1 ; 132(1): 8-43$

14. Peh WM, Kang ML. A pilot survey on an understanding of point of care bedside ultrasound (POCUS) among medical doctors in internal medicine: Exposure, perceptions, interest and barriers to training. Proc Singapore Healthc. 2017 Sep 21;27(2):85-95.

15. Olgers TJ, Ter Maaten JC. Point-of-care ultrasound curriculum for internal medicine residents: What do you desire? A national survey. BMC Med Educ. 2020 Jan 31;20(1):30.

16. Peng S, Micks T, Braganza D, Sue K, Woo M, Rogers P, et al. Canadian national survey of family medicine residents on point-of-care ultrasound training. Can Fam Physician. 2019 Dec;65(12):e523-30.

17. See KC, Ong V, Wong SH, Leanda R, Santos J, Taculod J, et al. Lung ultrasound training: curriculum implementation and learning trajectory among respiratory therapists. Intensive Care Med. 2016 Jan 1;42(1):63-71.

18. See KC, Ong V, Ng J, Tan RA, Phua J. Basic critical care echocardiography by pulmonary fellows: Learning trajectory and prognostic impact using a minimally resourced training model. Crit Care Med. 2014;42(10):2169-77.

19. Millington SJ, Hewak M, Arntfield RT, Beaulieu Y, Hibbert B, Koenig S, et al. Outcomes from extensive training in critical care echocardiography: Identifying the optimal number of practice studies required to achieve competency. J Crit Care. 2017 Aug;40:99-102.

20. Sewa DW, Anantham D. Overcoming ethical challenges of bedside medical education. Ann Acad Med Singapore. 2017;46(1):1-3.

21. Cunningham CT, Quan H, Hemmelgarn B, Noseworthy T, Beck $\mathrm{CA}$, Dixon E, et al. Exploring physician specialist response rates to web-based surveys. BMC Med Res Methodol. 2015;15(1):32.
Supplement 1. Online Survey Form
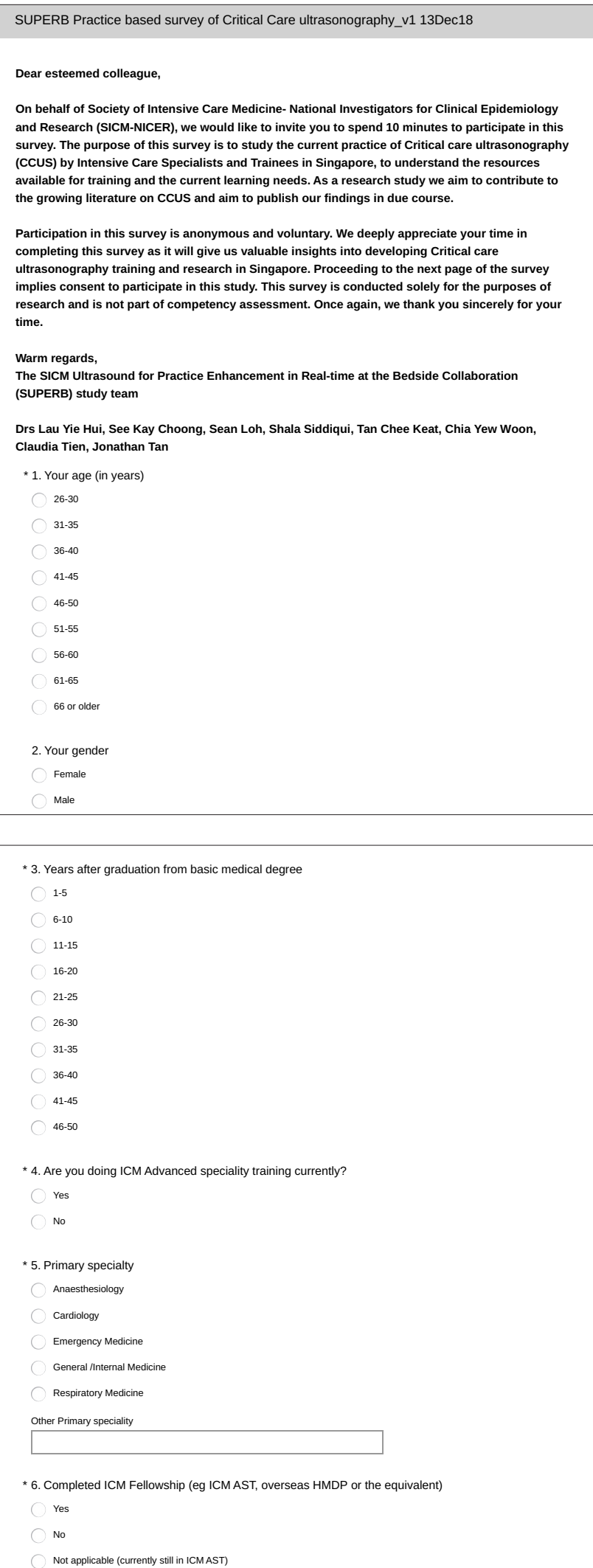

Copyright (C) 2020 Annals, Academy of Medicine, Singapore 

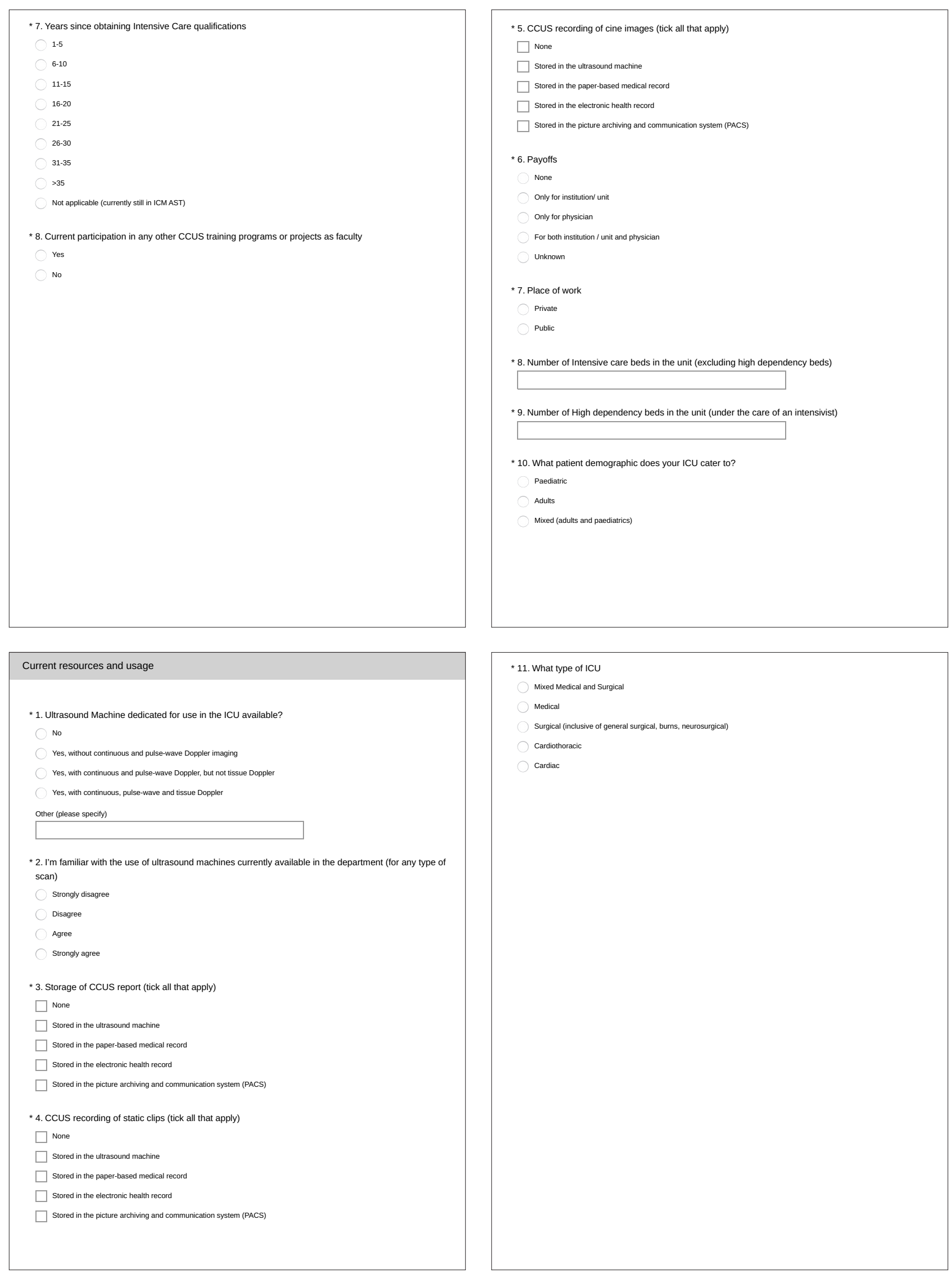

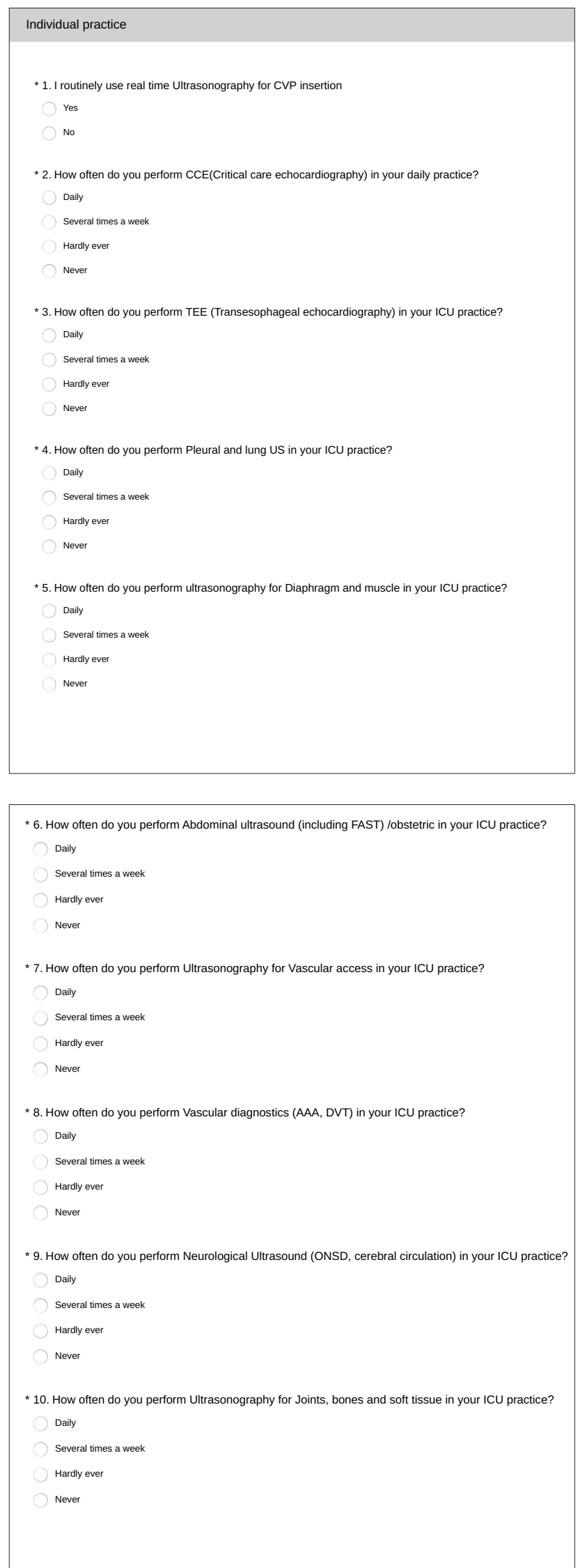

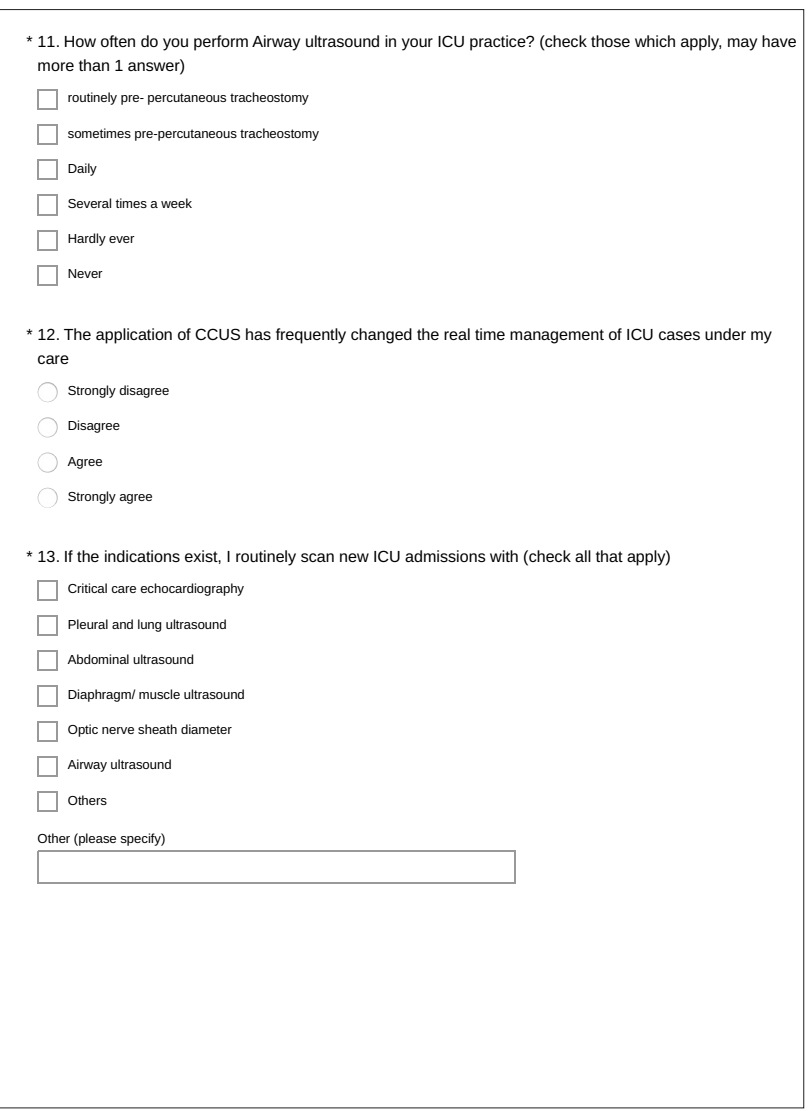

\section{Critical care ultrasound training}

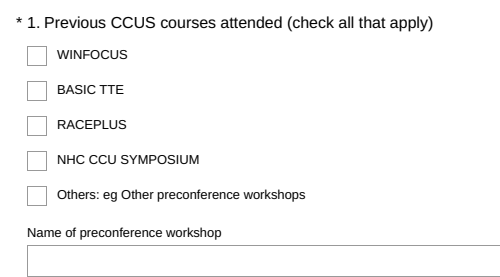

* 2. Formal* Echocardiography attachments / electives/ training (eg with Cardiology/ during ICM fellowship) of at least one month duration (*Formal= direct supervision for minimum number of scans, with assigned supervisor)

C. Yes

No

* 3. Received Formal* ultrasound training in (check those which apply) $\square$ TEE
$\square \operatorname{CCE}$ (Critical care echocardiography)
$\square$ Aimay
Pleural and lung ultrasound
$\square$ Diaphragm and muscle
$\square$ Abdominal/obstetric
$\square$ FAST
Vascular access
7 Vascular diagnostics (AAA, DVT)
Neurological (ONSD, cerebral circulation)
Joints, bones and soft tissue
$\square$ other (please specify) 

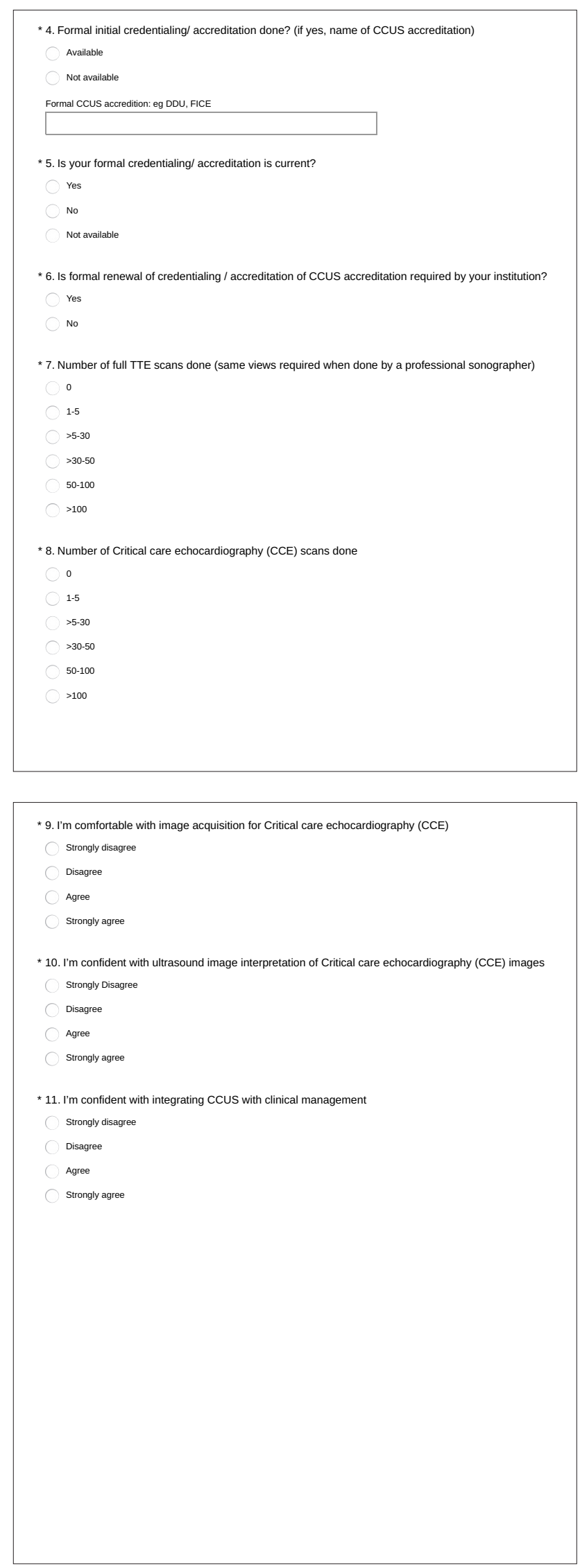

\section{Barriers}

* 1. What are the perceived obstacles when embarking on a formal training program for ccus currently?

(Check those which apply)

$\square$ Lack of supervisors

$\square$ Overseas (eg ASUM, BSE, EDEC) programs are costly

$\square$ Lack of ultrasound equipment

Lack of storage for clips and images

$\square$ Lack of time to scan

No need for competency assessment for management at the bedside

$\square$ Medico-legal implications

Inadequate knowledge

$\square$ Inadequate practice opportunities

$\square$ others

Other (please specity)

\section{Future practice needs assessment}

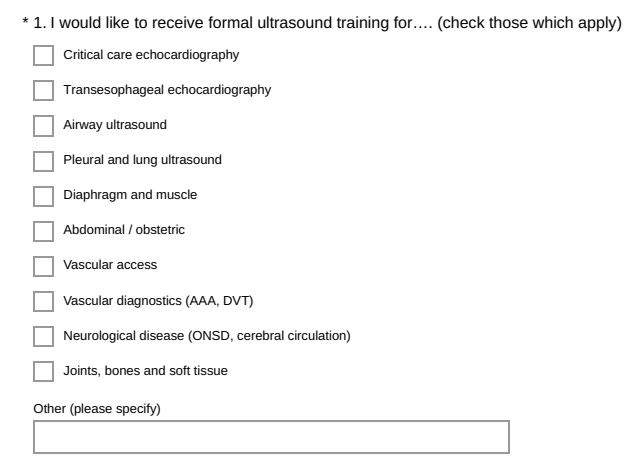



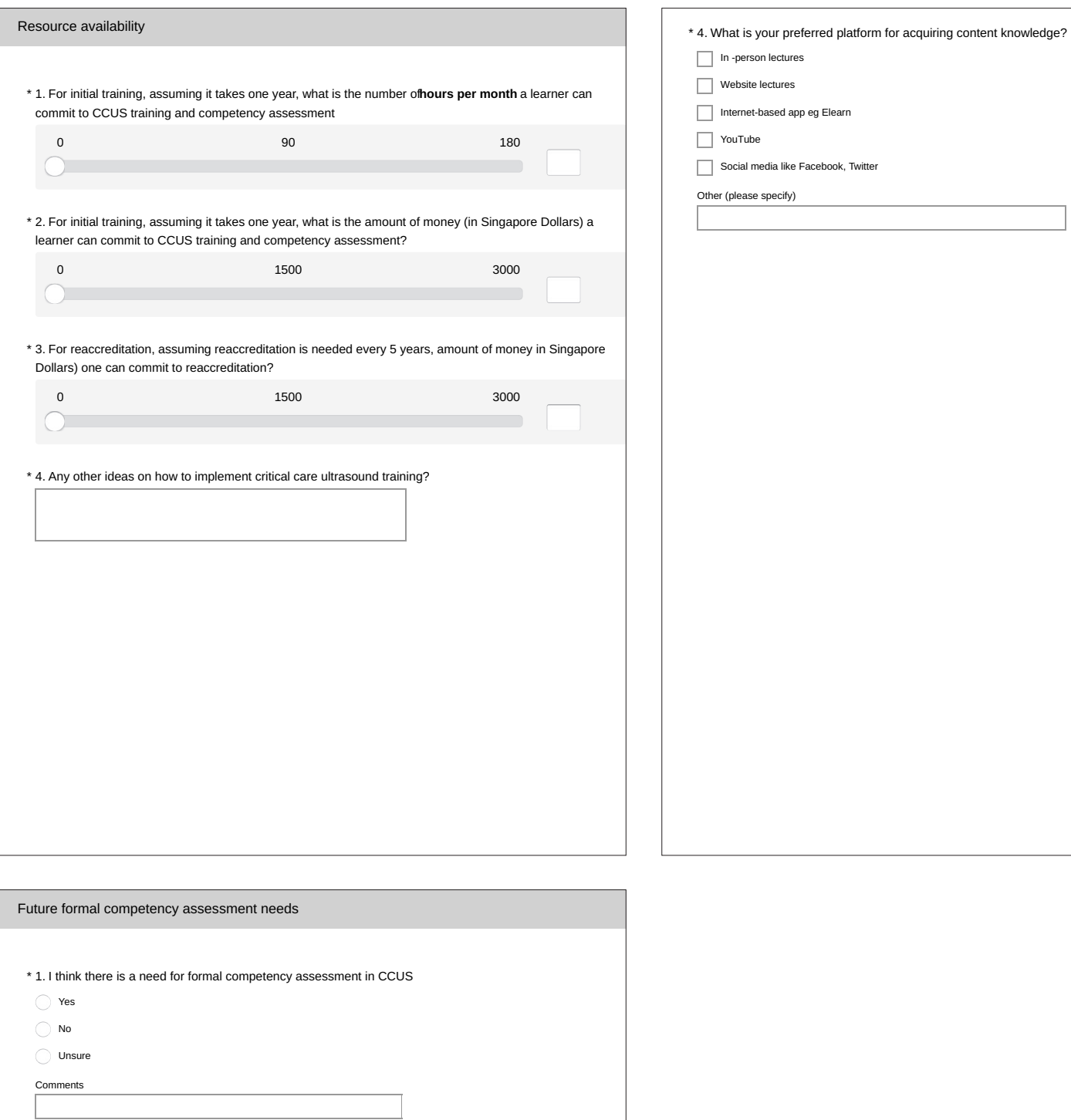

* 2.1 think there is a need for a local ccus training program

Strongly agree

Agree

Disagree

Strongly disagree

* 3 . I would like to receive formal competency assessment for (check those which apply)

$\square$ CCE Critical care echocardiography

$\square$ TEE Transesophageal echocardiography

$\square$ Pleural and lung ultrasound

$\square$ Diaphragm and muscle

$\square$ Abdominal/obstetric

$\square$ FAST

$\square$ Vascular access

$\square$ Vascular diagnostics (AAA, DVT)

$\square$ Neurological (ONSD, cerebral circulation)

$\square$ Joints, bones and soft tissue

$\square$ Airway 